\title{
Comparing long-term outcomes between endoscopic submucosal dissection (ESD) and endoscopic mucosal resection (EMR) for type II esophagogastric junction neoplasm
}

\author{
Yong Liu ${ }^{1}$, Shun $\mathrm{He}^{1}$, Yueming Zhang ${ }^{1}$, Lizhou Dou ${ }^{1}$, Xiao Liu ${ }^{1}$, Xinying Yu ${ }^{1}$, Ning Lu ${ }^{2}$, Liyan Xue ${ }^{2}$, \\ Guiqi Wang ${ }^{1}$
}

${ }^{1}$ Department of Endoscopy, National Cancer Center/National Clinical Research Center for Cancer/Cancer Hospital, Chinese Academy of Medical Sciences and Peking Union Medical College, Beijing, China; ${ }^{2}$ Department of Pathology, National Cancer Center/National Clinical Research Center for Cancer/Cancer Hospital, Chinese Academy of Medical Sciences and Peking Union Medical College, Beijing, China

Contributions: (I) Conception and design: Y Liu, L Xue, G Wang; (II) Administrative support: Y Liu, S He; (III) Provision of study materials or patients: Y Liu, S He, Y Zhang; (IV) Collection and assembly of data: Y Liu, X Liu, X Yu; (V) Data analysis and interpretation: Y Liu, L Xue; (VI) Manuscript writing: All authors; (VII) Final approval of manuscript: All authors.

Correspondence to: Guiqi Wang, MD, PhD. Department of Endoscopy, National Cancer Center/National Clinical Research Center for Cancer/ Cancer Hospital, Chinese Academy of Medical Sciences and Peking Union Medical College, 17 Panjiayuan, Chaoyang District, Beijing 100021, China. Email: wangguiq@126.com; Liyan Xue, MD, PhD. Department of Pathology, National Cancer Center/National Clinical Research Center for Cancer/Cancer Hospital, Chinese Academy of Medical Sciences and Peking Union Medical College, 17 Panjiayuan, Chaoyang District, Beijing 100021, China. Email: xueliyan2003@126.com.

Background: Endoscopic submucosal dissection (ESD) and endoscopic mucosal resection (EMR) are used to remove esophagogastric junction (EGJ) neoplasm. This study aimed to compare feasibility, safety, and effectiveness between ESD and EMR to help endoscopists choose treatment methods.

Methods: A total of 130 patients with EGJ neoplasm underwent endoscopic resection, including 52 patients with EMR and 78 patients with ESD. Cap-assisted EMR (EMRC) was performed with typical sequences. Larger lesions required removal in multiple pieces (i.e., piecemeal EMR). The ESD procedures were included that marking the periphery of the lesion, submucosa injected, circumferentially cutting and submucosal dissection. Resection time, adverse events, en bloc resection rate, R0 resection rate and recurrence rate were compared between the two groups.

Results: There were no significant differences in demographic characteristics or histopathological features between the two groups. Resection time was longer in the ESD group than in the EMR group (64.4 $\pm 33.9 v s$. $22.1 \pm 8.0$ minutes; $\mathrm{P}<0.01)$. Adverse events were more common in the ESD group than in the EMR group $(16.7 \%$ vs. $3.8 \%$; $\mathrm{P}=0.03)$, including bleeding (7.7\% vs. $3.8 \%)$, perforation $(5.1 \%$ vs. $0 \%)$ and stenosis $(5.1 \%$ vs. $0 \%)$. The en bloc resection rate and R0 resection rate were much higher in the ESD group than in the EMR group (98.7\% and $92.3 \%$ vs. $23.1 \%$ and $23.1 \%$, respectively; $\mathrm{P}<0.01$ ). The 5 -year overall survival rate and disease-free survival rate were $100 \%$ vs. $92.0 \%$ and $100 \%$ vs. $90.1 \%$ between the ESD and EMR groups, respectively (P=0.01 and $\mathrm{P}=0.01$ ). The 5 -year cancer-specific survival rate was $100 \%$ vs. $96.0 \%$ between the ESD and EMR groups $(\mathrm{P}=0.08)$. The recurrence rate was lower in the ESD group than in the EMR group $(0 \% v s .9 .6 \% ; \mathrm{P}=0.01)$.

Conclusions: ESD is an acceptable first-line endoscopic treatment for type II EGJ neoplasm, however, it is time-consuming and has a higher rate of adverse events. Furthermore, EMR is a safe and alternative technique, particularly when EMR could achieve en bloc resection.

Keywords: Esophagogastric junction neoplasm (EGJ neoplasm); long-term outcomes; endoscopic submucosal dissection (ESD); endoscopic mucosal resection (EMR)

Submitted May 26, 2020. Accepted for publication Nov 26, 2020.

doi: 10.21037/atm-20-4265

View this article at: http://dx.doi.org/10.21037/atm-20-4265 


\section{Introduction}

Esophagogastric junction (EGJ) neoplasm is a kind of gastrointestinal tumor, which located mainly at the distal esophagus and/or cardia. Endoscopic resection for EGJ neoplasm is difficult due to its narrow lumen and sharp angle (1). Endoscopic resection for EGJ neoplasm is a stomach-preserving technique and quality of life is better comparing with gastrectomy (2-5). Endoscopic resection technique includes endoscopic mucosal resection (EMR) and endoscopic submucosal dissection (ESD). The three major steps of EMR are injection, suction and resection. Variations of EMR include snare polypectomy, strip biopsy, cap-assisted EMR (EMRC), and EMR with ligation technique (EMRL). EMR is easy to perform and is associated with lower adverse events. EMR methods usually performed for the lesion less than $2 \mathrm{~cm}$ in diameter. Larger lesions required removal in multiple pieces. However, the main drawback of EMR is piece-meal resection which might lead to reconstructed specimen and residual tumor. The local recurrence or metachronous carcinoma rate after EMR ranges from $6 \%$ to $23 \%(6-8)$. ESD is regarded as a more difficult procedure to remove superficial gastrointestinal lesions. ESD enables the resection of even large lesions in a single piece. The main advantage of ESD is its excellent en bloc resection rate, curative resection rate, and low risk of local recurrence (8-10). However, ESD has a higher risk of adverse event, such as perforation and bleeding.

Type II EGJ neoplasms, according to Siewert's classification (11), refer to the cases in which the epicenter of the tumor is located within the area $1 \mathrm{~cm}$ above and $2 \mathrm{~cm}$ below the EGJ. Type II EGJ adenocarcinoma can be divided into Barrett's adenocarcinoma and gastric cancer. The occurrence and treatment of EGJ neoplasm differ between Western countries and East Asia. In Western countries, EGJ neoplasm cases are mainly adenocarcinoma in the long segment of the Barrett's esophagus, and EMR or EMR with radiofrequency ablation (RFA) is the main endoscopic treatment $(12,13)$. However, in East Asia, gastric cancer which defined as type II EGJ adenocarcinoma, is more common. Even Barrett's adenocarcinoma often arises from the short segment of the esophagus. In East Asia, ESD is the main endoscopic treatment (13-16). To the best of our knowledge, studies on endoscopic resection for type II EGJ neoplasm are limited. There is no study comparing long-term outcomes between ESD and EMR just for type II EGJ neoplasm $(13,17)$. Furthermore, whether the expanded criteria for endoscopic resection in early gastric cancer can be applied in type II EGJ neoplasm is still controversial. We performed a retrospective study to compare ESD with EMR based on feasibility, safety, and effectiveness for type II EGJ neoplasm to help endoscopists choose treatment methods and to determine the suitability of the expanded criteria. We present the following article in accordance with the STROBE reporting checklist (available at http://dx.doi. org/10.21037/atm-20-4265).

\section{Methods}

\section{Patients}

From October 2008 to February 2015, a total of 130 patients with early-stage type II EGJ neoplasm underwent endoscopic resection by GW. The inclusion criteria were as follows: (I) patients with Siewert type II EGJ neoplasm, which refers to a tumor with an epicenter located within the area $1 \mathrm{~cm}$ above and $2 \mathrm{~cm}$ below the EGJ (11); (II) patients with adenocarcinoma or high-grade glandular intraepithelial neoplasia (HGIN); and (III) patients who underwent endoscopic resection, EMR or ESD. The exclusion criteria were as follows: (I) patients with Siewert type I or III EGJ neoplasm; (II) patients who underwent surgery or combination therapy, such as endoscopic resection with argon plasma coagulation (APC); (III) patients with endoscopic ultrasound or computed tomography (CT) showing a progressive tumor or suspicious metastasis; (IV) patients with low-grade glandular intraepithelial neoplasia (LGIN); and (V) patients with squamous cell carcinoma or its precursor. From October 2008 to June 2013, 52 patients with early-stage EGJ neoplasm were treated by EMR. GW is experienced at endoscopic resection for early esophageal and gastric cancer. He had performed 31 esophageal ESD and 56 gastric ESD procedures before March 2013. As EGJ has been regarded as a difficult location for endoscopic treatment due to its narrow lumen and sharp angle, he began to perform ESD for type II EGJ neoplasm since 2013. With improvement in skills, from March 2013 to February 2015, 78 patients with early-stage EGJ neoplasm were treated by ESD. The trial was conducted in accordance with the Declaration of Helsinki (as revised in 2013). The study was approved by the Institutional Review Board of National Cancer Center/National Clinical Research Center for Cancer/Cancer Hospital, Chinese Academy of Medical Sciences and Peking Union Medical College (No. 19/242-2026) and informed consent was taken from all the patients. 


\section{EMR and ESD techniques}

As the EGJ is a difficult location for endoscopic treatment, we performed EMRC. The EMR procedures were as follows: the periphery of the lesion was marked with the tip of a snare, approximately $2 \mathrm{~mm}$ away from the lesion; diluted epinephrine $(1: 100,000)$ was injected into the submucosa; the lesion was drawn into the cap by suction, and the snare was closed snugly; the snared lesion was then released from the cap and resected $(18,19)$. Larger lesions required removal in multiple pieces (i.e., piecemeal EMR).

The ESD procedures were as follows: the periphery of the lesion was marked with a dual knife (KD-650L; Olympus Optical Co., Ltd., Tokyo, Japan), at least $5 \mathrm{~mm}$ away from the lesion, except on the oral side, where the marking was placed $1 \mathrm{~cm}$ from the squamocolumnar junction (SCJ) or tumor border (20); diluted epinephrine $(1: 100,000)$ was injected into the submucosa along the presumed cutting line; the mucosa surrounding the lesion was circumferentially cut with a dual knife (KD-650L) or an IT knife (KD-610L; Olympus Optical Co., Ltd.); and submucosal dissection of the connective tissue of the submucosa under the lesion was performed (21).

\section{Histopathological evaluation}

Specimens were flattened on a plate with thin needles and fixed in $10 \%$ formalin for 24 hours. Then, the specimens were cut into $2-\mathrm{mm}$ sections and embedded in paraffin. Five-micron sections were cut, mounted on glass slides and stained with hematoxylin and eosin. All slides were evaluated by two experienced gastrointestinal pathologists. The Vienna classification of gastrointestinal epithelial neoplasia was used for histopathologic evaluation (22). Histopathological evaluation included tumor size, differentiation type, depth of invasion, lateral and vertical margins, and the presence of lymphatic and/or vascular invasion. Submucosal invasion depth was measured from the deepest muscularis mucosa to the deepest tumor invasion in the submucosa (6). En bloc resection was defined as the removal of the lesion, including all markings, in one piece. Complete resection (R0 resection) was defined as en bloc resection with tumor-free lateral and vertical margins. Curative resection was defined using the expanded criteria for endoscopic resection for early gastric cancer as follows: en bloc resection, tumor-free lateral and vertical margins, no lymphatic or vascular invasion, and (I) tumor size $>2 \mathrm{~cm}$, histologically of differentiated type, pT1a, ulcerative findings (UL) (-); (II) tumor size $\leq 3 \mathrm{~cm}$, histologically of differentiated type, pT1a, UL(+); (III) tumor size $\leq 2 \mathrm{~cm}$, histologically of undifferentiated type, pT1a, UL(-); or (IV) tumor size $\leq 3 \mathrm{~cm}$, histologically of differentiated type, $\mathrm{pT} 1 \mathrm{~b}$ (sm1, <500 microns from the muscularis mucosae) (23-26).

\section{Follow-up}

Patients underwent endoscopy at 3, 6, 12 months and annually thereafter. In addition, patients underwent chest and abdominal CT annually. Patients who did not match the curative criteria and had not undergone surgery were advised to undergo endoscopy and CT scan every 6 months. Local recurrence was defined as a lesion with adenocarcinoma or HGIN located at the scar and occurred after the index endoscopic resection. Distant recurrence was defined as lymph node metastasis and organ metastasis that occurred after the index endoscopic resection during follow-up (6).

\section{Statistical analysis}

The primary outcome of the study is comparing the long-term outcome of ESD with that of EMR for type II EGJ neoplasm. The secondary outcome of the study is determining the suitability of the expanded criteria for endoscopic resection for early gastric cancer. Followup outcome data were collected in January 2020. We supplemented missing data by making phone calls or writing letters to the patients or the patients' local hospital. Two patients (3.8\%) in the EMR group and 1 patient (1.4\%) in the ESD group were lost to follow-up. Statistical analysis was performed with SAS statistical software, version 9.4 (SAS Institute Inc., Cary, NC, USA). Continuous variables with a normal distribution were described by the mean $( \pm \mathrm{SD})$. For variables with a skewed distribution the median (IQR) was used. Differences between treatment groups were compared with Student's $t$-test or the Mann-Whitney test for continuous variables. Categorical data were compared with the Chi-square test. All tests were two-tailed, and $\mathrm{P}<0.05$ was considered statistically significant.

Adverse events include perforation, postoperative bleeding and postoperative stenosis. Adverse events rate was compared with the Chi-square test between the EMR group and ESD group. The efficacy outcome assessment include procedure time, en bloc resection rate, $\mathrm{R} 0$ resection rate curative resection rate and long-term outcome. Procedure time defined as the time between the delineation of the 
lesion periphery and the end of the endoscopic resection procedure, including removal of specimens and treatment of intraoperative bleeding and perforation. Procedure time was described by the mean $\left({ }_{ \pm} \mathrm{SD}\right)$ and analyzed with Student's $t$-test. We perform the receiver operating curve (ROC) analysis to identify the best tumor size cutoff for the optimal en bloc resection rate in the EMR group. Longterm outcome include the 5-year overall survival rate, cancer-specific survival rate and disease-free survival rate. We analyzed a subgroup long-term outcome with tumor size under $2 \mathrm{~cm}$ between the EMR group and ESD group. We also divided the EMR group into an en bloc group (12 patients) and a piece-meal group (40 patients) to analysis long-term outcome. The log-rank test was used in the Kaplan-Meier method. The overall survival, cancer-specific survival and disease-free survival analyses were conducted using Kaplan-Meier curves. The 5 -year overall survival rate, cancer-specific survival rate and disease-free survival rate were calculated using the life table method. All tests were two-tailed, and $\mathrm{P}<0.05$ was considered statistically significant.

\section{Results}

\section{Demographic characteristics and histopathological features}

From October 2008 to February 2015, a total of 130 patients with early-stage type II EGJ neoplasm underwent endoscopic resection by GW. The demographic characteristics and histopathological features of all patients are summarized in Table 1. The EMR group consisted of

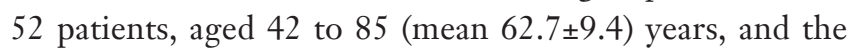
ESD group consisted of 78 patients, aged 44 to 78 (mean $63.2 \pm 7.4$ ) years. The EMR group comprised 43 men (83\%) and 9 women (17\%), and the ESD group comprised 66 men $(85 \%)$ and 12 women (15\%). The median maximum diameter of the lesion was $18 \mathrm{~mm}$ (IQR: 14-21) and $20 \mathrm{~mm}$ (IQR: 14-32) in the EMR group and ESD group, respectively. There were no significant differences between the two groups regarding age, sex, median maximum diameter of the lesions, tumor morphology, histological type or tumor depth $(\mathrm{P}>0.05)$.

\section{Adverse events}

Adverse events in the two groups are summarized in Table 2. No perforation occurred in the EMR group, but perforation occurred in 4 patients $(5.1 \%)$ in the ESD group.
All perforations were closed with endoclips. Postoperative bleeding occurred in 2 patients $(3.8 \%)$ treated by EMR and in 6 patients $(7.7 \%)$ treated by ESD. All bleeding events were treated in the same endoscopic session using coagulation forceps. No postoperative stenosis occurred in the EMR group, but postoperative stenosis occurred in 4 patients (5.1\%) in the ESD group. All cases of stenosis were managed with balloon dilation. In conclusion, 2 patients $(3.8 \%)$ in the EMR group and 13 patients $(16.7 \%)$ in the ESD group experienced adverse events. One patient from the ESD group experienced simultaneous perforation and postoperative bleeding. There was a significant difference between the two groups with respect to the overall rate of adverse events $(\mathrm{P}=0.025)$. The mean of hospital stay period was $6.5(\mathrm{SD}: \pm 2.8)$ and $7.9(\mathrm{SD}: \pm 1.8)$ days in the EMR group and ESD group, respectively. There was a significant difference between the two groups with respect to the hospital stay period $(\mathrm{P}<0.01)$. In addition, no death occurred during hospitalization period, which was due to complications.

\section{Procedural outcomes and resection specimens}

In the EMR group, a median of 3 (IQR: 2-4) resections were required to remove the lesion. Twelve lesions were resected en bloc by endoscopy, and the en bloc resection rate was $23.1 \%$. In the ESD group, 77 lesions were resected en bloc by endoscopy, and the en bloc resection rate was $98.7 \%$. There was a statistically significant difference between the two groups in the en bloc resection rate $(\mathrm{P}<0.01)$. The average resection time was $22.1(\mathrm{SD}: \pm 8.0)$ minutes in the EMR group and $64.4(\mathrm{SD}: \pm 33.9)$ minutes in the ESD group, indicating a statistically significant difference between the two groups $(\mathrm{P}<0.01)$. A significant difference was found between the two groups with respect to the maximum diameter of the specimen, with values of 26.2 (SD: $\pm 8.2) \mathrm{mm}$ in the EMR group vs. $54.5(\mathrm{SD}: \pm 15.1) \mathrm{mm}$ in the ESD group $(\mathrm{P}<0.01)$. Details of the histologic findings are summarized in Table 1.

\section{Treatment outcomes}

In the EMR group, the rates of en bloc resection and R0 resection were both $23.1 \%$. The ROC analysis suggested that the tumor size for the optimal en bloc resection rate in the EMR group was $15 \mathrm{~mm}$, with an area under the curve equal to 0.952 (sensitivity: $91.7 \%$, specificity: $90 \%$ ), as shown in Figure 1. Ten cases showed margin positivity, deep 
Table 1 Demographic characteristics and histopathological features

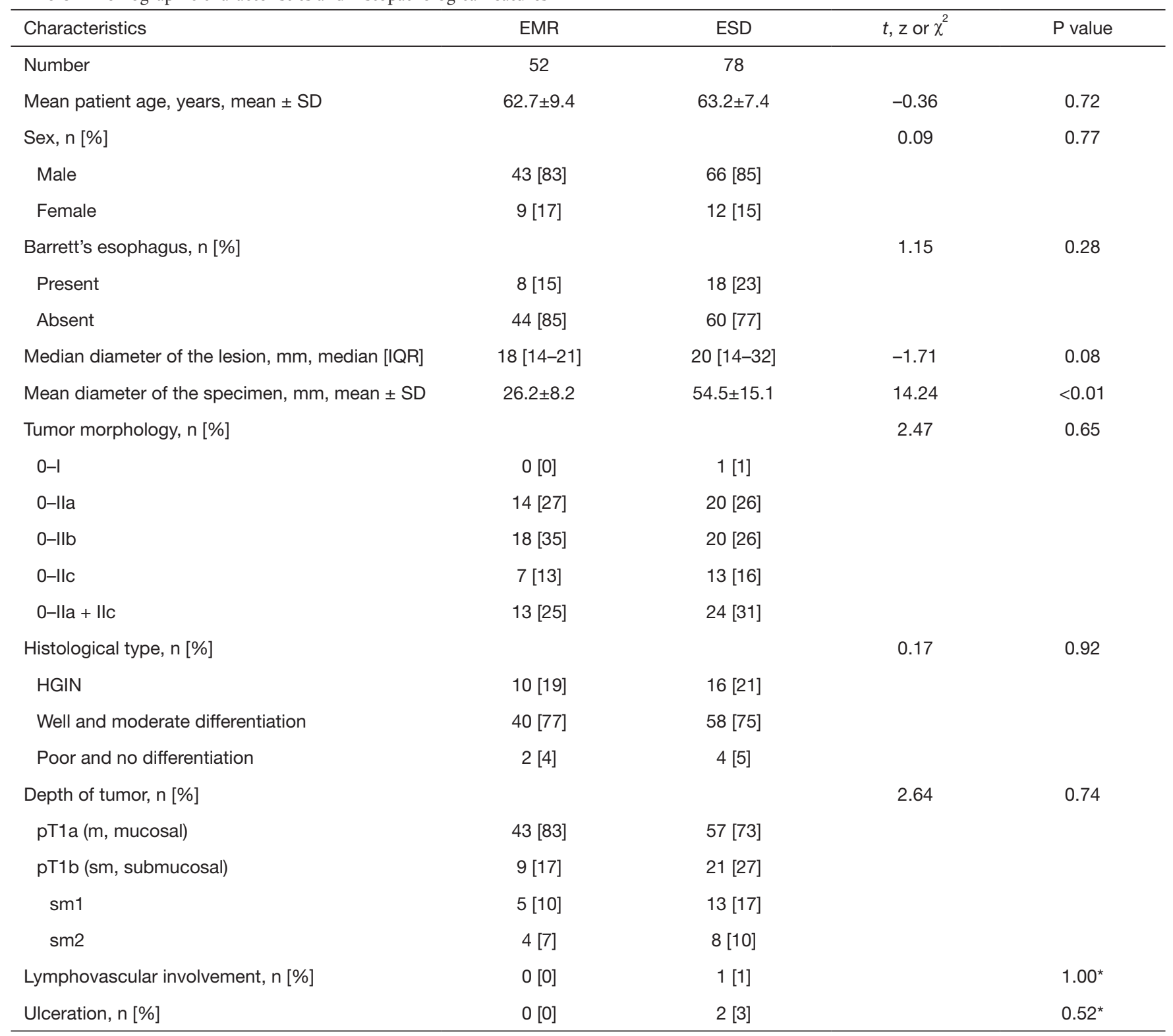

*, Fisher's exact test. EMR, endoscopic mucosal resection; ESD, endoscopic submucosal dissection; HGIN, high-grade glandular intraepithelial neoplasia.

Table 2 Adverse events

\begin{tabular}{lccc}
\hline Adverse events & EMR & ESD & $\chi^{2}$ \\
\hline Total, $\mathrm{n}(\%)$ & $2(3.8)$ & $13(16.7)$ & 5.02 \\
Postoperative bleeding, $\mathrm{n}(\%)$ & $2(3.8)$ & $6(7.7)$ & \\
Perforation, $\mathrm{n}(\%)$ & $0(0.0)$ & $4(5.1)$ & \\
Stenosis, $\mathrm{n}(\%)$ & $0(0.0)$ & $4(5.1)$ & \\
\hline
\end{tabular}

In the ESD group, one patient experienced both perforation and postoperative bleeding. EMR, endoscopic mucosal resection; ESD, endoscopic submucosal dissection. 

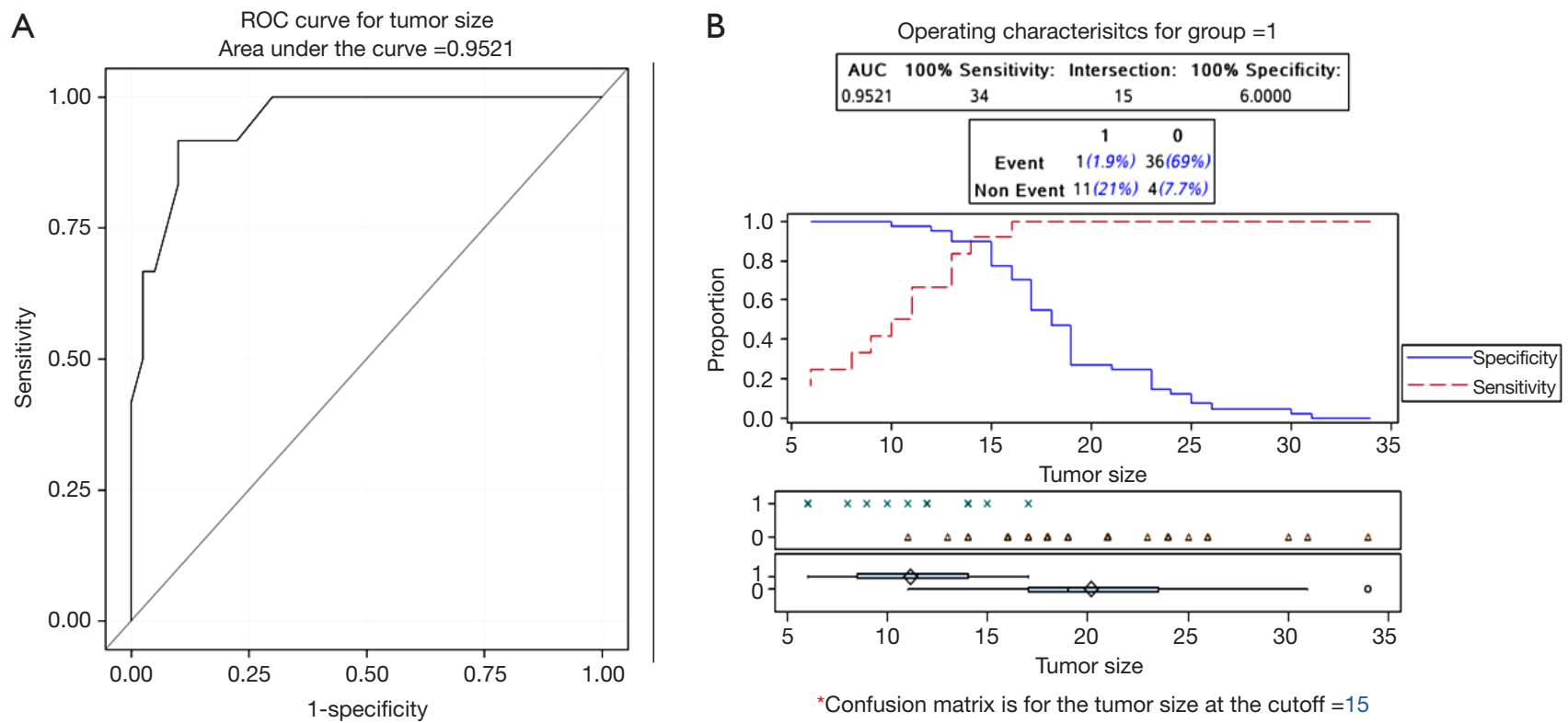

Figure 1 ROC and best tumor size cutoff. (A) ROC; (B) best tumor size cutoff of the ROC curve. The ROC analysis suggested that the tumor size for the optimal en bloc resection rate in the EMR group was $15 \mathrm{~mm}$, with an area under the curve equal to 0.952 (sensitivity: 91.7\%, specificity: 90\%). ROC, receiver operating curve; EMR, endoscopic mucosal resection.

submucosal invasion and poor differentiation. The vertical margin of the specimen was positive in four patients, and among them, two showed submucosal invasion deeper than $500 \mu \mathrm{m}$ and one showed lateral margin positivity. Of the four patients with positive vertical margins, two underwent surgery, one underwent chemotherapy because of a surgical history of distal gastrectomy and poor physical condition, and one refused to undergo additional therapy because of poor physical condition. The lateral margin of the specimen indicated HGIN in three cases. Because the specimen was reconstructed in the EMR group and the result might have been inaccurate, all three patients in the EMR group chose to undergo follow-up instead of further treatment. In addition, two cases simply showed submucosal invasion deeper than $500 \mu \mathrm{m}$, and one case was histopathologically defined as having poor differentiation. These patients also refused to undergo surgery and chose follow-up.

In the ESD group, the rates of en bloc resection, R0 resection and curative resection were $98.7 \%, 92.3 \%$ and $75.6 \%$, respectively. Nineteen patients were considered to have undergone noncurative resection. The vertical margin of the specimen was positive in five patients: three of them also showed submucosal invasion deeper than $500 \mu \mathrm{m}$, one of them had poor differentiation, and one of them showed lymphovascular involvement. Of the five patients with positive vertical margins, three underwent surgery and two refused to undergo surgery. The lateral margin of one case indicated HGIN. The pathological result of this patient indicated intramucosal cancer, and he did not undergo further treatment because of the burning effect and lack of risk of lymph node metastasis (27). In addition, five cases showed submucosal invasion deeper than $500 \mu \mathrm{m}$, three cases were histopathologically defined as the undifferentiated type, and five cases showed a tumor size larger than $30 \mathrm{~mm}$ with minute submucosal invasion. These patients also refused to undergo surgery and chose follow-up.

\section{Follow-up outcomes}

The follow-up outcomes of the two groups are summarized in Table 3. The 5-year overall survival rate and diseasefree survival rate were significantly different between the ESD and EMR groups, with values of $100 \%$ vs. $92.0 \%$ and $100 \%$ vs. $90.1 \%$, respectively $(\mathrm{P}=0.01$ and $\mathrm{P}=0.01)$. The 5 -year cancer-specific survival rate was $100 \%$ vs. $96.0 \%$ in the ESD and EMR groups ( $\mathrm{P}=0.08)$. The median followup time in the ESD group and EMR group was 67 (IQR: 63.8-70.0) and 87.5 (IQR: 80-94.5) months, respectively $(\mathrm{P}<0.01)$. During the follow-up period, local recurrence 
Table 3 Long-term outcomes during follow-up

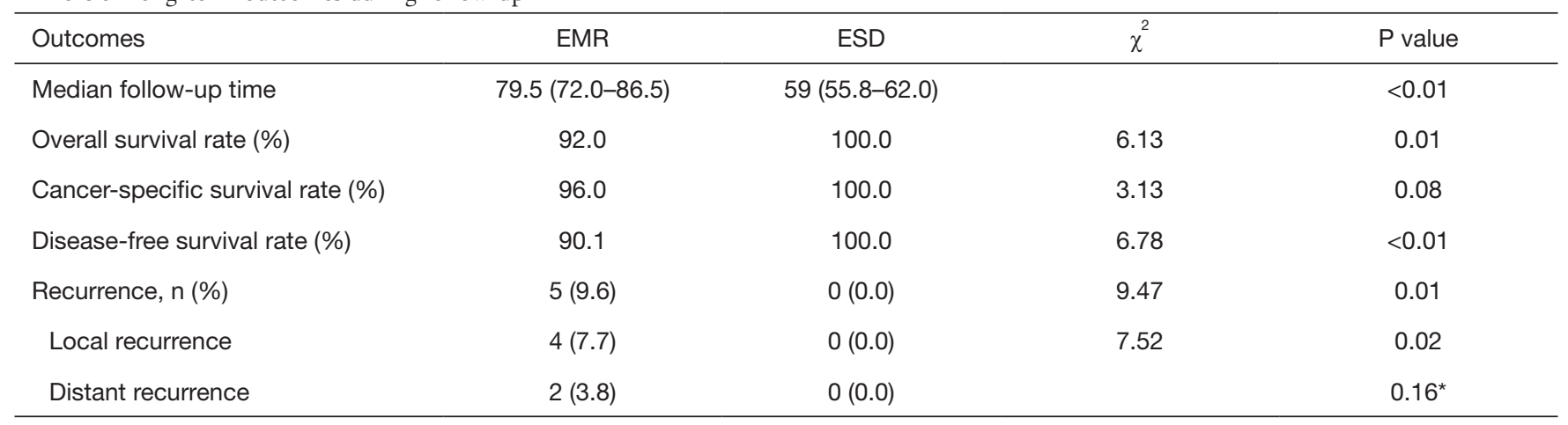

*, Fisher's exact test. EMR, endoscopic mucosal resection; ESD, endoscopic submucosal dissection.

Table 4 Clinicopathologic characteristics of patients with recurrence after EMR

\begin{tabular}{|c|c|c|c|c|c|c|c|}
\hline Case & Size (mm) & Morphology & Histology & Depth & Resection status & Lateral margin & Vertical margin \\
\hline 2 & 16 & $0-I I b$ & Well-differentiated & p-T1a & Piecemeal & $(-)$ & $(-)$ \\
\hline 3 & 31 & $0-$-lla & Well-differentiated & $\mathrm{p}-\mathrm{T} 1 \mathrm{~b}(700 \mu \mathrm{m})$ & Piecemeal & $(-)$ & $(+)$ \\
\hline 4 & 18 & $0-I I a+I I c$ & Well-differentiated & $\mathrm{p}-\mathrm{T} 1 \mathrm{a}$ & Piecemeal & $(+)$ & $(-)$ \\
\hline
\end{tabular}

EMR, endoscopic mucosal resection; HGIN, high-grade glandular intraepithelial neoplasia.

Table 5 Clinicopathologic characteristics of recurrent tumors after EMR

\begin{tabular}{cccccccc}
\hline Case & $\begin{array}{c}\text { Time to local } \\
\text { recurrence (months) }\end{array}$ & $\begin{array}{c}\text { Time to distant } \\
\text { metastasis (months) }\end{array}$ & Size $(\mathrm{mm})$ & Morphology & Histology & $\begin{array}{c}\text { Additional } \\
\text { treatment }\end{array}$ & Arognosis \\
\hline 1 & 8 & NA & 4 & $0-$ Ilb & HGIN & APC & Alive \\
2 & 7 & NA & 7 & 0-Ila & pT1a & Surgery & Died of other disease \\
3 & NA & 10 & NA & NA & NA & Chemotherapy & Died of the disease \\
4 & 23 & NA & 12 & 0-Ila & pT1a & ESD & Died of other disease \\
5 & 18 & 18 & 41 & Ulcerating mass & cT3 & Chemotherapy & Died of the disease \\
\hline
\end{tabular}

EMR, endoscopic mucosal resection; HGIN, high-grade glandular intraepithelial neoplasia; ESD, endoscopic submucosal dissection; APC, argon plasma coagulation.

occurred in $4(7.7 \%)$ patients, and distant recurrence was observed in $2(3.8 \%)$ patients in the EMR group. One patient experienced both local and distant recurrence. The primary tumor clinicopathologic characteristics of local and distant recurrence after EMR are shown in Table 4. The primary tumors in all five patients were treated by the piecemeal method. Nevertheless, none of the patients experienced local or distant recurrence in the ESD group. Significant differences between the two groups were observed in terms of local and distant recurrence. The clinicopathologic characteristics of locally and distally recurrent tumors after EMR are shown in Table 5. All patients who experienced local recurrence or distant metastasis in the EMR group underwent additional therapy. The recurring lesions of patients 1 and 4 were cured by additional endoscopic treatment. Patient 2 underwent additional surgical resection. However, patients 2 and 4 died of hypopharyngeal cancer and bladder carcinoma, 
A
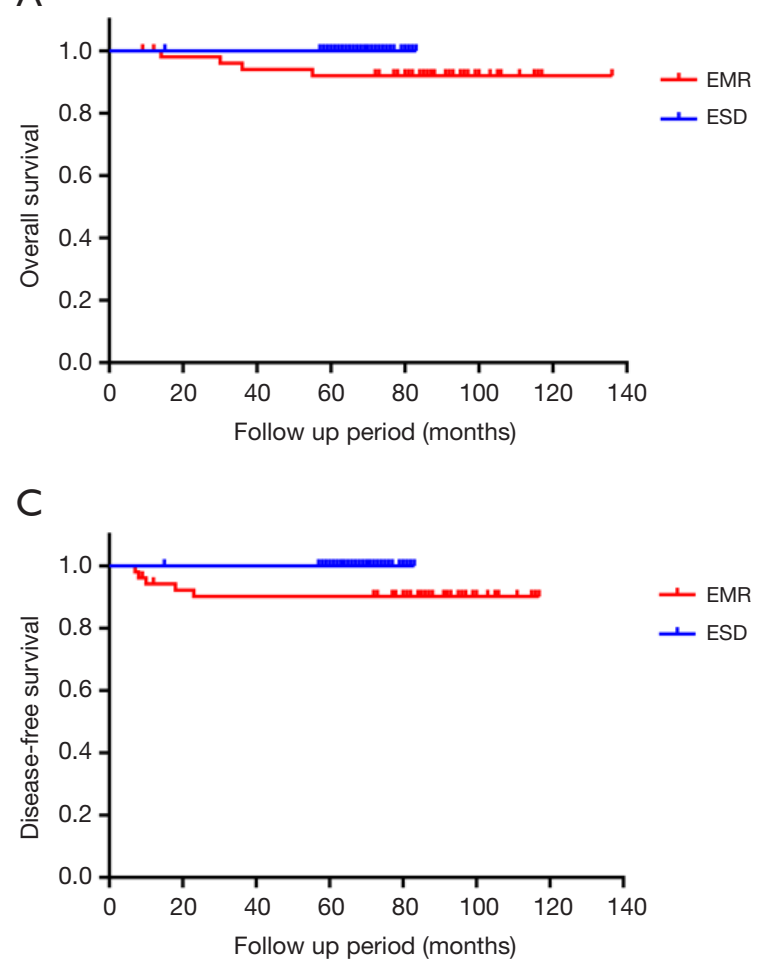

B

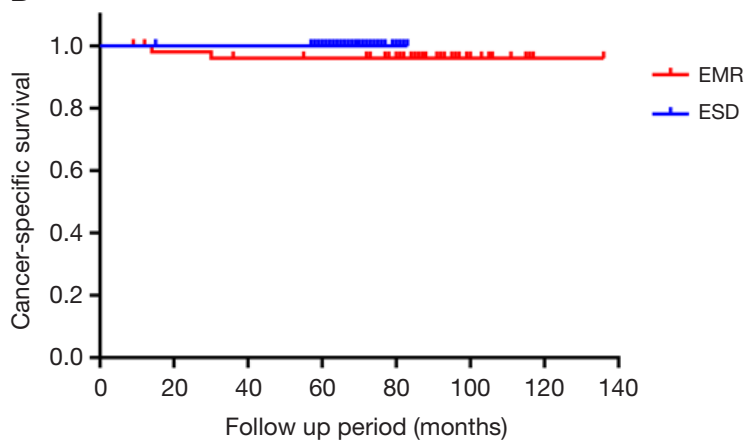

Figure 2 The 5-year follow-up outcome. (A) Five-year overall survival Kaplan-Meier curve; (B) 5-year cancer-specific survival Kaplan-Meier curve; (C) 5-year disease-free survival Kaplan-Meier curve. The 5-year overall survival rate and disease-free survival rate were higher in the ESD group than in the EMR group for type II EGJ neoplasm (100\% vs. 92.0\% and 100\% vs. 90.1\%, respectively; P=0.01 and P=0.01). The 5 -year cancer-specific survival rate was higher in the ESD group than in the EMR group; nevertheless, there were no significant differences between the two groups (100\% vs. 96.0\%, $\mathrm{P}=0.08)$. Although the follow-up time in the ESD group was shorter than that in the EMR group, the follow-up outcomes were better in the ESD group than in the contemporaneous EMR group. ESD, endoscopic submucosal dissection; EMR, endoscopic mucosal resection; EGJ, esophagogastric junction.

respectively. Patients 3 and 5 underwent chemotherapy but still died of the disease at 14 and 30 months after EMR. The primary tumors of two patients with distant recurrence showed submucosal invasion deeper than $500 \mu \mathrm{m}$. In the ESD group, one patient with lateral margin HGIN was not offered further endoscopic intervention, and two patients with vertical margin-positive disease refused salvage surgery. Nonetheless, no local or distant recurrence was found during the follow-up period. The 5-year overall survival Kaplan-Meier curve, cancer-specific survival Kaplan-Meier curve and disease-free survival Kaplan-Meier curve of two groups are summarized in Figure 2.

We analyzed a subgroup with tumor size under $2 \mathrm{~cm}$ between the EMR group and ESD group (33 patients and 42 patients, respectively). During the follow-up period, local recurrence occurred in $3(9.1 \%)$ patients, and none of patients experienced distant recurrence in the EMR group. None of the patients experienced local or distant recurrence in the ESD group. The recurrence rate was lower in the ESD group than in the EMR group ( $0 \%$ vs. $9.1 \%$, respectively; $\mathrm{P}=0.02$ ). The 5 -year overall survival Kaplan-Meier curve, cancer-specific survival Kaplan-Meier curve and disease-free survival Kaplan-Meier curve of these groups are summarized in Figure 3.

We also divided the EMR group into an en bloc group (12 patients) and a piece-meal group (40 patients). During the follow-up period, local recurrence occurred in $4(7.7 \%)$ patients, and distant recurrence was observed in $2(3.8 \%)$ patients in the piecemeal group. One patient experienced both local and distant recurrence. Nevertheless, none of the patients experienced local or distant recurrence in the en bloc group. The recurrence rate and local recurrence 
A

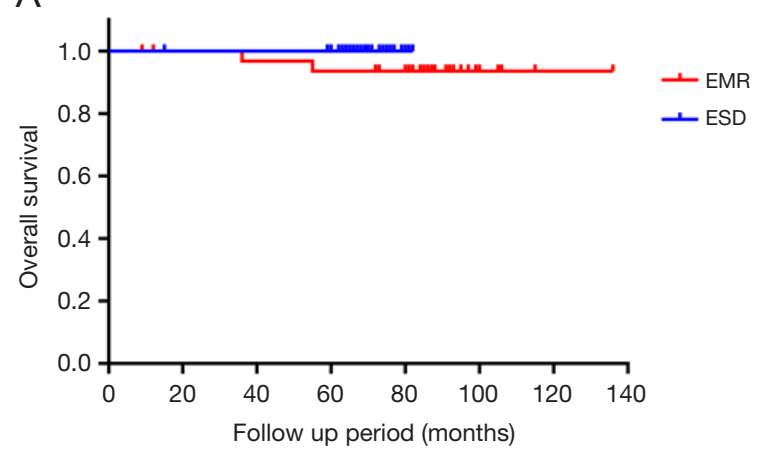

C

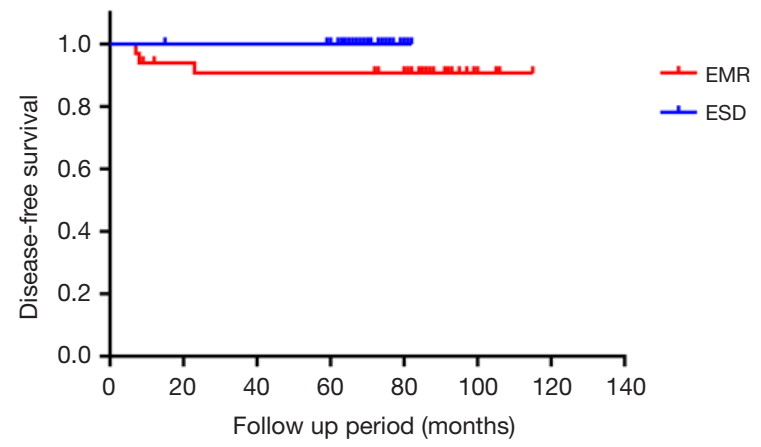

B

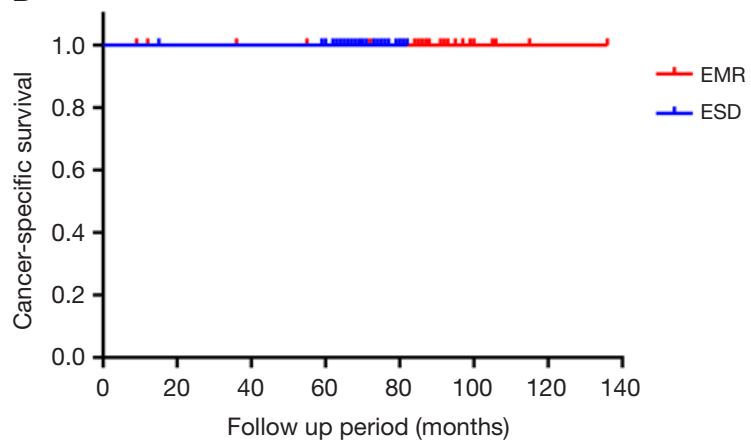

Figure 3 The 5-year follow-up outcome between the ESD than the EMR group with tumor size under $2 \mathrm{~cm}$. (A) Five-year overall survival Kaplan-Meier curve; (B) 5-year cancer-specific survival Kaplan-Meier curve; (C) 5-year disease-free survival Kaplan-Meier curve. The 5 -year overall survival rate and disease-free survival rate was higher in the ESD than in the EMR group with tumor size under $2 \mathrm{~cm}$ (100\% vs. $93.6 \%$, and $100 \%$ vs. $90.5 \%$, respectively; $\mathrm{P}=0.12$ and $\mathrm{P}=0.07)$. The 5 -year cancer-specific survival rate was same in the ESD than in the EMR group with tumor size under $2 \mathrm{~cm}(100 \%$ vs. 100\%; $\mathrm{P}=1.00)$. Although there was no statistically significant difference between two groups, the follow-up outcomes of 5 -year overall survival rate and disease-free survival rate were better in the ESD than in the EMR group with tumor size under $2 \mathrm{~cm}$. ESD, endoscopic submucosal dissection; EMR, endoscopic mucosal resection.

rate was lower in the en bloc group than in the piecemeal group ( $0 \%$ vs. $12.5 \%, 0 \%$ vs. $10 \%$, respectively; $\mathrm{P}=0.10$ and $\mathrm{P}=0.14)$. The 5-year overall survival Kaplan-Meier curve, cancer-specific survival Kaplan-Meier curve and diseasefree survival Kaplan-Meier curve of these groups are summarized in Figure 4.

\section{Discussion}

To the best of our knowledge, this is the first retrospective study comparing ESD with EMR just for type II EGJ neoplasm. We included 130 patients with early-stage type II EGJ neoplasm who underwent endoscopic resection. We divided these patients into two groups according to the procedure: the EMR group and the ESD group. There were no significant differences between the two groups in terms of age, sex, median maximum diameter of the lesions, tumor morphology, or histological type.

The ESD group showed a longer resection time and a higher rate of adverse events than did the EMR group. In our opinion, the increased resection time and rate of adverse events reflect the higher technical complexity of ESD. The major drawback of ESD is the long procedure time, particularly in difficult locations, such as the EGJ (13). In addition, the difference in the size of the resected specimen may have led to a longer resection time and higher rates of adverse events in the ESD group. Although ESD resulted in a higher rate of adverse events, all adverse events were managed by endoscopy. No serious adverse events that required surgical treatment or blood transfusion were encountered. On the other hand, compared with ESD, EMR is a minimally invasive and easy-to-learn procedure. Thus, EMR might be suitable for older patients or patients with poor physical condition who cannot endure a long 

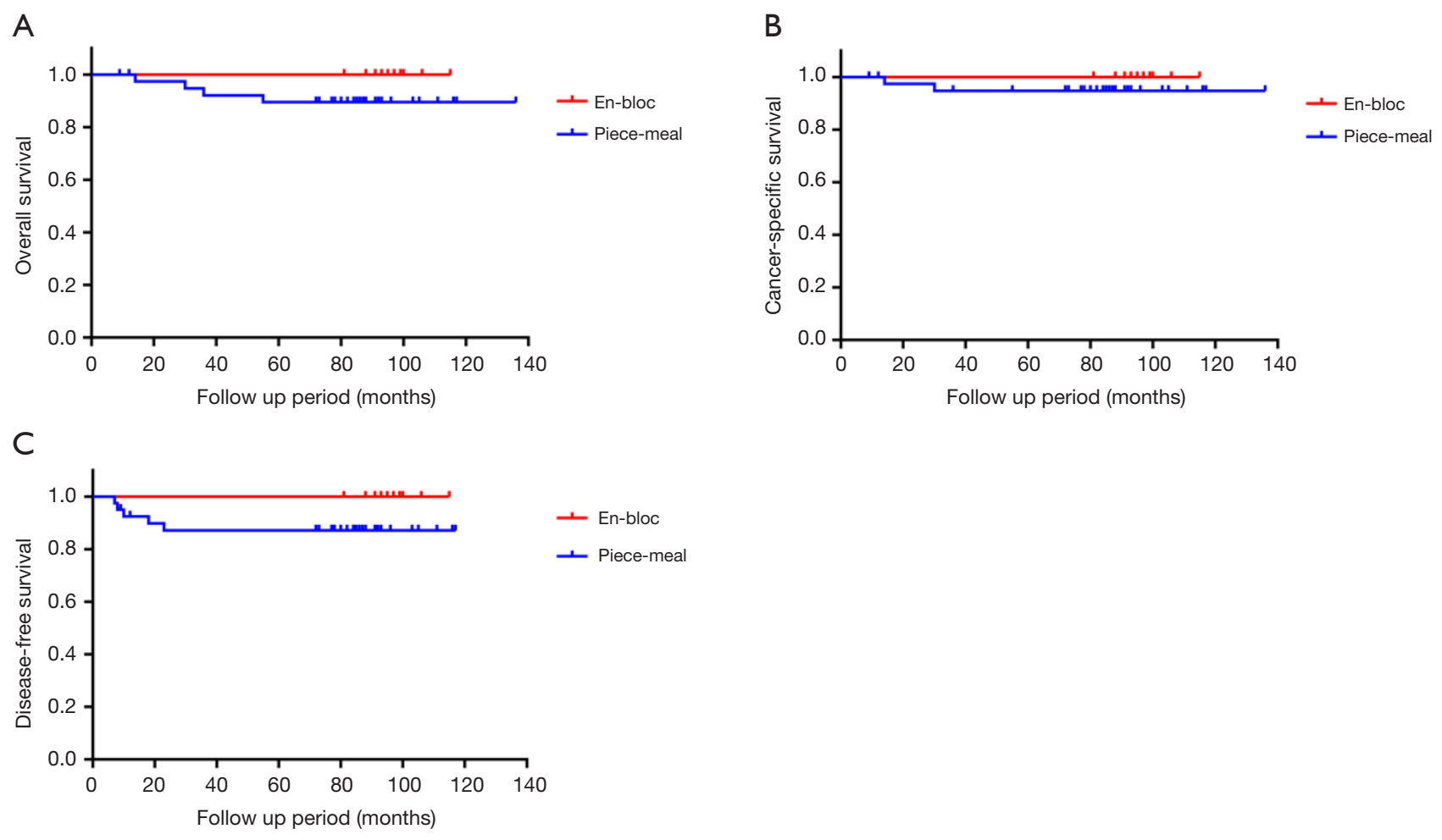

Figure 4 The 5-year follow-up outcome between en bloc group and piece-meal group in EMR group. (A) Five-year overall survival KaplanMeier curve; (B) 5-year cancer-specific survival Kaplan-Meier curve; (C) 5-year disease-free survival Kaplan-Meier curve. The 5-year overall survival rate, cancer-specific survival rate and disease-free survival rate was higher in the en bloc group than in the piece-meal group (100\% vs. $89.5 \%, 100 \%$ vs. $94.8 \%$, and $100 \%$ vs. $87.1 \%$, respectively; $\mathrm{P}=0.25, \mathrm{P}=0.42$ and $\mathrm{P}=0.20)$. Although there was no statistically significant difference between two groups, the follow-up outcomes were better in the en bloc group than in the piece-meal group. EMR, endoscopic mucosal resection.

procedure time.

The ESD group showed a much higher en bloc resection rate and $\mathrm{R} 0$ resection rate than the EMR group (98.7\% and $92.3 \%$ vs. $23.1 \%$ and $23.1 \%$, respectively; $\mathrm{P}<0.05$ ). In contrast with EMR, ESD enables the resection of even large lesions in a single piece (28). Although the EGJ has been regarded as a difficult location for endoscopic treatment, several studies have reported en bloc resection rates of $90-100 \%$ for ESD in EGJ cancer $(6,8,24,29)$. Although we performed EMR with cap, the en bloc resection rate was still low in the treatment of EGJ dysplasia. The major drawback of EMR is the lower en bloc resection rate than ESD. The en bloc resection rate in our study $(23.1 \%)$ was even lower than that in cases of early gastric cancer treated with EMR (40.9-72.2\%) (30). One reason is that type II EGJ neoplasm might extend beyond the cardia, including the angle of His (20). The difficult location may lead to an obviously lower en bloc resection rate. On the other hand, the median diameter of the lesion was $18 \mathrm{~mm}$ in the EMR group. Larger lesions often require piecemeal resection. In East Asia, the pathologic types of EGJ adenocarcinoma are different from those of Western countries, as most of Chinese EGJ neoplasms are cardiac adenocarcinoma. Even Barrett's adenocarcinoma often arises from the short-segment Barrett's esophagus $(12,13,16)$. For such type II EGJ neoplasms with difficult locations, our study is the first to demonstrate that EMR may be likely to achieve en bloc resection, when the tumor size is smaller than $15 \mathrm{~mm}$.

Differences in the size of the resected specimen are related to the delineation of the lesion periphery. The marked border in EMR is approximately $2 \mathrm{~mm}$ away from the lesion, but in ESD, it is at least $5 \mathrm{~mm}$ away from the lesion, which leads to a difference in the size of the resected specimen. According to histopathologic evaluation after EMR, we found that EGJ neoplasm might invade the lower 
esophagus, even if the lower esophagus mucosa looks normal by endoscopy. The histopathologic results of one patient in the EMR group who underwent additional surgery because of vertical margin positivity showed adenocarcinoma invading underneath the normal esophageal squamous epithelium and the lack of residual tumor at the previous endoscopic resection site. With piecemeal resection and reconstructed specimens, the results of the lateral margin might be inaccurate. EGJ adenocarcinoma might invade the mucosal stroma under the normal esophageal squamous epithelium, which defined as subsquamous carcinoma extension $(14,15,20)$. If subsquamous carcinoma extension is not visible by endoscopy, the periphery of the oral side cannot be accurately determined (20). Whitelight endoscopy, magnifying endoscopy with narrow-band imaging (NBI) and dye spraying with acetic acid or indigocarmine are used to assess the periphery of the oral side. These studies also indicated several endoscopic signs of subsquamous carcinoma extension, such as slight elevation, a change in mucosal color, the formation of small holes and annular vessels (20,31,32). Conversely, since most cases of subsquamous carcinoma extension are reported to be less than $1 \mathrm{~cm}$ in size, oral safety margins placed $1 \mathrm{~cm}$ from the SCJ or endoscopic signs of subsquamous carcinoma extension are useful in ESD for EGJ adenocarcinoma $(17,20)$. In the ESD group, there was no case with a positive horizontal margin on the oral side, indicating that oral margins placed $1 \mathrm{~cm}$ from the SCJ or endoscopic signs of subsquamous carcinoma extension are safe.

The 5-year overall survival rate and disease-free survival rate were higher in the ESD group than in the EMR group for type II EGJ neoplasm (100\% vs. 92.0\% and $100 \%$ vs. $90.1 \%$, respectively; $\mathrm{P}<0.05)$. The 5 -year cancer-specific survival rate was higher in the ESD group than in the EMR group; nevertheless, there were no significant differences between the two groups $(100 \% v s$. $96.0 \%, \mathrm{P}>0.05)$. During the follow-up period, the ESD group showed significantly lower rates of local and distant recurrence than the EMR group. Complete resection reduces the residual recurrence rate during follow-up (30). EMR methods usually performed for the lesion less than $2 \mathrm{~cm}$ in diameter. We analyzed a subgroup with tumor size under $2 \mathrm{~cm}$ between the EMR group and ESD group (33 patients and 42 patients, respectively). There was no statistically significant difference between two groups in the aspect of 5-year overall survival rate, 5-year cancer-specific survival rate and disease-free survival rate. But the followup outcomes of 5-year overall survival rate and disease-free survival rate were better in the ESD than in the EMR group with tumor size under $2 \mathrm{~cm}$, which were shown in Figure 3. However, EMR often involves fragmentation of the lesion, which makes the histologic evaluation difficult (8), and piecemeal EMR may cause minor remnants of neoplastic tissue to be retained (28). In 2006, Ono et al. reported that local recurrence was observed more often with piecemeal EMR than with en bloc resection for the treatment of early gastric cancer $(8,33)$. In our study, we observed similar outcomes: the local recurrence rate was lower in the en bloc group than in the piecemeal group $(0 \%$ vs. $10 \% ; \mathrm{P}>0.05)$. Although there was no statistically significant difference between two groups, the follow-up outcomes were better in the en bloc group than in the piece-meal group, which were shown in Figure 4. Therefore, treatment of EGJ neoplasm by EMR is recommended for the lesion, which less than $2 \mathrm{~cm}$ in diameter and could be removed en bloc. In contrast, treatment of EGJ neoplasm by ESD resulted in high en bloc resection rates $(6,8,10,23)$, making histologic evaluation more accurate, especially for the lateral margin. In the ESD group, one patient with lateral margin positivity and two patients with vertical margin positivity did not receive additional treatment, but no local or distant recurrence was found during the follow-up period. In these three patients, the only noncurative factor was the positive resection margin based on the expanded ESD criteria. The patient with lateral margin positivity showed the presence of HGIN at the positive margin, with a length of $2 \mathrm{~mm}$. Furthermore, the length of the positive vertical margin in the two patients was less than $0.5 \mathrm{~mm}$. The reason for the absence of recurrence in these three patients might be the burning effect and lack of risk of lymph node metastasis. Several studies have reported residual tumors in only 15.4$65 \%$ of patients with lateral margin positivity in the surgical specimen (34-38). In the case of noncurative resection in patients with only one positive lateral resection margin, additional endoscopic treatment or observation could be an alternative strategy (39). Although two patients with vertical margin positivity did not show recurrence, additional surgery should be encouraged.

Type II EGJ adenocarcinoma can be divided into Barrett's adenocarcinoma and gastric cancer. However, whether EGJ neoplasm can be treated as an esophageal cancer or as a gastric cancer is controversial (23). The main argument is that minute submucosal invasion is defined as an invasion of 200 or $500 \mu \mathrm{m}$. An overview of surgical series reporting the risk of lymph node metastasis in gastric and EGJ tumors has shown that the risk of lymph node 
metastasis in minimal submucosal invasion ( $\mathrm{sm} 1$ or $<500 \mu \mathrm{m}$ from the muscularis mucosa) was low (0-14\%), but that for deeper submucosal invasion was high, ranging from $21 \%$ to $46 \%(8,40,41)$. Two patients with distant recurrence in the EMR group showed submucosal invasion deeper than $500 \mu \mathrm{m}$. In conclusion, piecemeal EMR and submucosal invasion deeper than $500 \mu \mathrm{m}$ may have led to worse longterm prognosis in the EMR group than in the ESD group. From the previous study and our present results, curative resection, which is defined according to the expanded criteria for endoscopic resection for early gastric cancer, may be applicable.

Our study has several limitations. First, this was a retrospective study and a single institution. Second, the follow-up time was different between the two groups. Because ESD for type II EGJ neoplasm is an emerging technique since 2013 at our hospital while EMR is a relatively mature technique, the follow-up time for the EMR group was longer than that for the ESD group. Third, some patients undergoing noncurative resection chose follow-up, although surgery is standard care for such patients. Nevertheless, most patients with noncurative resection fulfilled the complete resection criteria, and there was no evidence of metastasis. After surgical consultation, these patients chose to undergo follow-up.

In conclusion, with higher en bloc resection and R0 resection rates and better long-term outcome during followup, ESD is an acceptable first-line endoscopic treatment for type II EGJ neoplasm. However, ESD is more timeconsuming and has a higher rate of adverse events, which requires highly skilled endoscopists. Furthermore, EMR is a minimally invasive and easy-to-learn procedure for removing EGJ neoplasm, which requires low technique skill and might be suitable for older patients or patients with poor physical condition who cannot endure a long procedure time. EMR has similar long-term outcome to that of ESD, when EMR could achieve en bloc resection. Thus, EMR might be more suitable for the tumor size is smaller than $15 \mathrm{~mm}$. In addition, the expanded criteria for endoscopic resection for early gastric cancer, may be applicable for type II EGJ neoplasm.

\section{Acknowledgments}

Funding: This work was supported by the National Key R\&D Program of China (No. 2016YFC1302801), Sanming Project of Medicine in Shenzhen (No. SZSM201911008), and CAMS Innovation Fund for Medical Sciences (CIFMS),
(No. 2016-I2M-1-001, 2017-I2M-1-001, 2019-I2M-2-004).

\section{Footnote}

Reporting Checklist: The authors have completed the STROBE reporting checklist. Available at http://dx.doi. org/10.21037/atm-20-4265

Data Sharing Statement: Available at http://dx.doi. org/10.21037/atm-20-4265

Conflicts of Interest: All authors have completed the ICMJE uniform disclosure form (available at http://dx.doi. org/10.21037/atm-20-4265). The authors have no conflicts of interest to declare.

Ethical Statement: The authors are accountable for all aspects of the work in ensuring that questions related to the accuracy or integrity of any part of the work are appropriately investigated and resolved. The trial was conducted in accordance with the Declaration of Helsinki (as revised in 2013). The study was approved by the Institutional Review Board of National Cancer Center/ National Clinical Research Center for Cancer/Cancer Hospital, Chinese Academy of Medical Sciences and Peking Union Medical College (No. 19/242-2026) and informed consent was taken from all the patients.

Open Access Statement: This is an Open Access article distributed in accordance with the Creative Commons Attribution-NonCommercial-NoDerivs 4.0 International License (CC BY-NC-ND 4.0), which permits the noncommercial replication and distribution of the article with the strict proviso that no changes or edits are made and the original work is properly cited (including links to both the formal publication through the relevant DOI and the license). See: https://creativecommons.org/licenses/by-nc-nd/4.0/.

\section{References}

1. Kakushima N, Yahagi N, Fujishiro M, et al. Efficacy and safety of endoscopic submucosal dissection for tumors of the esophagogastric junction. Endoscopy 2006;38:170-4.

2. Gotoda T. Endoscopic resection of early gastric cancer. Gastric Cancer 2007;10:1-11.

3. Pyo JH, Lee H, Min BH, et al. Long-term outcome of endoscopic resection vs. surgery for early gastric cancer: a non-inferiority-matched cohort study. Am J Gastroenterol 
2016;111:240-9.

4. Gong EJ, Kim DH, Ahn JY, et al. Comparison of longterm outcomes of endoscopic submucosal dissection and surgery for esophagogastric junction adenocarcinoma. Gastric Cancer 2017;20:84-91.

5. Pyo JH, Lee H, Min YW, et al. Indication for endoscopic treatment based on the risk of lymph node metastasis in patients with Siewert type II/III early gastric cancer. Gastric Cancer 2018;21:672-9.

6. Abe S, Ishihara R, Takahashi H, et al. Long-term outcomes of endoscopic resection and metachronous cancer after endoscopic resection for adenocarcinoma of the esophagogastric junction in Japan. Gastrointest Endosc 2019;89:1120-8.

7. Giovannini M, Bories E, Pesenti C, et al. Circumferential endoscopic mucosal resection in Barrett's esophagus with high-grade intraepithelial neoplasia or mucosal cancer. Preliminary results in 21 patients. Endoscopy 2004;36:782-7.

8. Hirasawa K, Kokawa A, Oka H, et al. Superficial adenocarcinoma of the esophagogastric junction: longterm results of endoscopic submucosal dissection. Gastrointest Endosc 2010;72:960-6.

9. Yoshinaga S, Gotoda T, Kusano C, et al. Clinical impact of endoscopic submucosal dissection for superficial adenocarcinoma located at the esophagogastric junction. Gastrointest Endosc 2008;67:202-9.

10. Park CH, Kim EH, Kim HY, et al. Clinical outcomes of endoscopic submucosal dissection for early stage esophagogastric junction cancer: a systematic review and meta-analysis. Dig Liver Dis 2015;47:37-44.

11. Siewert JR, Stein HJ. Classification of adenocarcinoma of the oesophagogastric junction. Br J Surg 1998;85:1457-9.

12. Bennett C, Vakil N, Bergman J, et al. Consensus statements for management of Barrett's dysplasia and early-stage esophageal adenocarcinoma, based on a Delphi process. Gastroenterology 2012;143:336-46.

13. Komeda Y, Bruno M, Koch A. EMR is not inferior to ESD for early Barrett's and EGJ neoplasia: An extensive review on outcome, recurrence and complication rates. Endosc Int Open 2014;2:E58-64.

14. Oyama T. Diagnostic strategies of superficial Barrett's esophageal cancer for endoscopic submucosal dissection. Dig Endosc 2013;25 Suppl 1:7-12.

15. Goda K, Singh R, Oda I, et al. Current status of endoscopic diagnosis and treatment of superficial Barrett's adenocarcinoma in Asia-Pacific region. Dig Endosc 2013;25 Suppl 2:146-50.
16. Sun Q, Huang Q, Feng AN, et al. Columnar-lined esophagus in Chinese patients with proximal gastric carcinomas. J Dig Dis 2013;14:22-8.

17. Nagami Y, Ominami M, Otani K, et al. Endoscopic Submucosal Dissection for Adenocarcinomas of the Esophagogastric Junction. Digestion 2018;97:38-44.

18. Soetikno RM, Gotoda T, Nakanishi Y, et al. Endoscopic mucosal resection. Gastrointest Endosc 2003;57:567-79.

19. May A, Gossner L, Behrens A, et al. A prospective randomized trial of two different endoscopic resection techniques for early stage cancer of the esophagus. Gastrointest Endosc 2003;58:167-75.

20. Nagami Y, Machida H, Shiba M, et al. Clinical efficacy of endoscopic submucosal dissection for adenocarcinomas of the esophagogastric junction. Endosc Int Open 2014;2:E15-20.

21. Gotoda T, Yamamoto H, Soetikno RM. Endoscopic submucosal dissection of early gastric cancer. J Gastroenterol 2006;41:929-42.

22. Schlemper RJ, Riddell RH, Kato Y, et al. The Vienna classification of gastrointestinal epithelial neoplasia. Gut 2000;47:251-5.

23. Yamada M, Oda I, Nonaka S, et al. Long-term outcome of endoscopic resection of superficial adenocarcinoma of the esophagogastric junction. Endoscopy 2013;45:992-6.

24. Imai K, Kakushima N, Tanaka M, et al. Validation of the application of the Japanese curative criteria for superficial adenocarcinoma at the esophagogastric junction treated by endoscopic submucosal dissection: a long-term analysis. Surg Endosc 2013;27:2436-45.

25. Ono H, Yao K, Fujishiro M, et al. Guidelines for endoscopic submucosal dissection and endoscopic mucosal resection for early gastric cancer. Dig Endosc 2016;28:3-15.

26. Japanese Gastric Cancer Association. Japanese gastric cancer treatment guidelines 2014 (ver. 4). Gastric Cancer 2017;20:1-19.

27. Hoteya S, Matsui A, Iizuka T, et al. Comparison of the clinicopathological characteristics and results of endoscopic submucosal dissection for esophagogastric junction and non-junctional cancers. Digestion 2013;87:29-33.

28. Neuhaus H, Wirths K, Schenk M, et al. Randomized controlled study of EMR versus endoscopic submucosal dissection with a water-jet hybrid-knife of esophageal lesions in a porcine model. Gastrointest Endosc 2009;70:112-20.

29. Omae M, Fujisaki J, Horiuchi Y, et al. Safety, efficacy, and long-term outcomes for endoscopic submucosal dissection 


\section{Page 14 of 14}

of early esophagogastric junction cancer. Gastric Cancer 2013;16:147-54.

30. Lian J, Chen S, Zhang Y, et al. A meta-analysis of endoscopic submucosal dissection and EMR for early gastric cancer. Gastrointest Endosc 2012;76:763-70.

31. Omae M, Fujisaki J, Shimizu T, et al. Magnifying endoscopy with narrow-band imaging findings in the diagnosis of Barrett's esophageal adenocarcinoma spreading below squamous epithelium. Dig Endosc 2013;25 Suppl 2:162-7.

32. Takeuchi M, Uedo N. Endoscopic detection of superficial esophagogastric junction adenocarcinoma. Dig Endosc 2017;29 Suppl 2:37-8.

33. Ono H. Early gastric cancer: diagnosis, pathology, treatment techniques and treatment outcomes. Eur J Gastroenterol Hepatol 2006;18:863-6.

34. Lee HJ, Jang YJ, Kim JH, et al. Clinical outcomes of gastrectomy after incomplete EMR/ESD. J Gastric Cancer 2011;11:162-6.

35. Jung H, Bae JM, Choi MG, et al. Surgical outcome after incomplete endoscopic submucosal dissection of gastric cancer. Br J Surg 2011;98:73-8.

36. Yoon H, Kim SG, Choi J, et al. Risk factors of residual or

Cite this article as: Liu Y, He S, Zhang Y, Dou L, Liu X, Yu X, Lu N, Xue L, Wang G. Comparing long-term outcomes between endoscopic submucosal dissection (ESD) and endoscopic mucosal resection (EMR) for type II esophagogastric junction neoplasm. Ann Transl Med 2021;9(4):322. doi: 10.21037/atm-20-4265

\section{Liu et al. Comparing ESD with EMR for type II EGJ neoplasm}

recurrent tumor in patients with a tumor-positive resection margin after endoscopic resection of early gastric cancer. Surg Endosc 2013;27:1561-8.

37. Hwang JJ, Park KJ, Park YS, et al. A scoring system for patients with a tumor-positive lateral resection margin after endoscopic resection of early gastric cancer. Surg Endosc 2016;30:2751-8.

38. Lee JH, Lee JH, Kim KM, et al. Clinicopathological factors of multiple lateral margin involvement after endoscopic submucosal dissection for early gastric cancer. Surg Endosc 2015;29:3460-8.

39. Jeon MY, Park JC, Hahn KY, et al. Long-term outcomes after noncurative endoscopic resection of early gastric cancer: the optimal time for additional endoscopic treatment. Gastrointest Endosc 2018;87:1003-13.e2.

40. Vieth M, Rosch T. Endoscopic mucosal resection and the risk of lymph-node metastases: indications revisited? Endoscopy 2006;38:175-9.

41. Westerterp M, Koppert LB, Buskens CJ, et al. Outcome of surgical treatment for early adenocarcinoma of the esophagus or gastro-esophageal junction. Virchows Arch 2005;446:497-504. 